\title{
Inferindo traços de personalidade com base em aplicativos móveis
}

\author{
Chaubert da Silva Ferreira ${ }^{1}$, Leonardo Júnio A. dos Santos Figueiredo ${ }^{1}$, \\ Fabrício A. Silva ${ }^{1}$, Thais R. M. Braga Silva ${ }^{1}$ \\ ${ }^{1}$ Universidade Federal de Viçosa (UFV) - Campus Florestal \\ Rodovia LMG 818, km 06, s/n, Florestal - MG, 35690-000 \\ \{chaubert.ferreira, leonardo.j.alves, fabricio.asilva, thais.braga\}@ufv.br
}

\begin{abstract}
Resumo. Conhecer o perfil de clientes é uma forma eficiente de oferecer serviços melhores e mais personalizados, aumentar a satisfação e, com isso, manter os mesmos engajados com a empresa. Um item muito relevante do perfil é a personalidade do usuário, que pode ser dividida em cinco traços distintos. Porém, inferir a qual traço um usuário pertence não é uma tarefa trivial. Neste trabalho, é investigado se os traços de personalidade podem ser inferidos com base nas categorias dos aplicativos instalados no dispositivo móvel de um usuário. Foram criados modelos de classificação e regressão, e os resultados são promissores, sendo possível identificar os traços com boa assertividade.

Abstract. Knowing the customers profile is an effective way to offer better and more personalized services, increase satisfaction, and keep them engaged with the company. A very relevant item of the profile is the user's personality that can be divided into five distinct traits. However, inferring which trait a user belongs to is not a trivial task. This work investigates whether we can infer personality traits based on the categories of applications installed on a user's mobile device. We create classification and regression models, and the results are promising, making it possible to identify the traits with good assertiveness.
\end{abstract}

\section{Introdução}

Conhecer os traços de personalidade de um usuário é importante pois ajuda a resumir, prever e explicar a conduta de um indivíduo [Silva and Nakano 2011]. Com esse conhecimento, empresas conseguem melhorar seus produtos e serviços, de forma que seus clientes se sintam mais satisfeitos e, consequentemente, mantenham a fidelidade e ajudem a melhorar sua visibilidade. Além disso, é possível criar e enriquecer um perfil de usuário, utilizado em sistemas de recomendação, identificação de churning, entre outros.

Porém, extrair os traços de personalidade de um indivíduo é uma tarefa complexa. Vários trabalhos já vem buscando resolver esse problema de diversas formas, sendo que muitas vezes usam de mecanismos invasivos que demandam informações sensíveis dos usuários. Em relação à extração dos traços de personalidade de usuários de serviços móveis, algumas soluções requerem que o usuário seja monitorado constantemente, exigindo um comprometimento do mesmo, e muitas vezes invadindo a sua privacidade.

A hipótese deste trabalho é que é possível extrair os traços de personalidade dos usuários com base nas categorias de seus aplicativos instalados com precisão considerável, sem que haja a necessidade de invadir a privacidade do usuário, exigir que o 
usuário responda a questionários extensos e que se comprometa a continuamente contribuir para a pesquisa. O objetivo do trabalho é validar essa hipótese e, para isso, serão coletados dados de aplicativos instalados de usuários voluntários, assim como as respostas a algumas perguntas referentes à sua personalidade. Assim, as respostas são utilizadas apenas para a criação dos modelos preditivos.

Um diferencial deste trabalho em relação aos existentes é que necessita apenas dos aplicativos instalados, o que não demanda nenhuma permissão extra do dispositivo [M. C. Grace and Sadeghi 2012]. Dessa forma, o usuário se beneficiará pois não terá de fornecer informações sensíveis, e empresas podem utilizar campanhas de marketing personalizadas em larga escala, pois a lista de aplicativos instalados é um dado mais fácil de se obter. Assim, foram desenvolvidos modelos preditivos de classificação e de regressão. Os resultados mostraram que foi possível classificar um usuário em termos de seus traços de personalidade com uma precisão considerável utilizando apenas as categorias dos aplicativos instalados.

Este trabalho está organizado da seguinte forma. Na Seção 2 são apresentados os conceitos e estudos relacionados. A Seção 3 descreve o aplicativo desenvolvido para a coleta dos dados, e uma caracterização inicial dos mesmos. Em seguida, na Seção 4 é feita uma análise preliminar dos dados, e são explicados os modelos preditivos e os seus resultados. Por fim, as conclusões e os trabalhos futuros são apresentados na Seção5

\section{Referencial Teórico}

Para o entendimento deste estudo, primeiramente é necessário apresentar quais são os traços de personalidade de referência, chamados de Big Five [McCrae and Jr. 1999]:

- Agradabilidade (AGR): Pessoas agradáveis, são gentis, confiáveis, tolerantes e mais propensas a ajudar aos outros;

- Aberto a Novas Experiências (ANE): Pessoas abertas a novas experiências, são pessoas criativas, de mente aberta, independentes e sempre estão procurando por diferentes experiências;

- Conscienciosidade (CON): Indivíduos deste traço são bem organizados, possuem autocontrole, são cuidadosos, persistentes e confiáveis;

- Estabilidade Emocional (EME): Pessoas emocionalmente estáveis são bem humoradas, não costumam sentir ansiedade, não são nervosas, possuem autoconfiança e tendem a não ter uma reação negativa diante de diferentes situações da vida;

- Extroversão (EXT): Pessoas extrovertidas, são comumente pessoas que gostam de se comunicar, ativas socialmente.

Uma das formas de estimar os traços de personalidade é utilizando o questionário TIPI (Ten-Item Personality Inventory) [Gosling et al. 2003]. Para calcular quantitativamente os traços de personalidade, é necessário responder a dez perguntas do TIPI de acordo com cada conceito, e depois fazer os cálculos correspondentes para cada traço, conforme ilustra a Tabela 1

Para calcular a nota do usuário em determinado traço de personalidade, é necessário somar a resposta de uma pergunta com o reverso de outra resposta complementar e calcular a média. Para isso, há um mapeamento entre os valores; caso o usuário tenha respondido com o valor 7 em uma pergunta, este valor será mapeado para o valor 1. Caso 
Tabela 1. Questionário TIPI (Adaptado de [Gosling et al. 2003])

(a) Valores dos Conceitos

\begin{tabular}{|lc|}
\hline Conceito & Valor \\
\hline Discordo Totalmente & 1 \\
\hline $\begin{array}{l}\text { Discordo Moderada- } \\
\text { mente }\end{array}$ & 2 \\
\hline Discordo um Pouco & 3 \\
\hline $\begin{array}{l}\text { Não discordo nem con- } \\
\text { cordo }\end{array}$ & 4 \\
\hline Concordo um pouco & 5 \\
\hline $\begin{array}{l}\text { Concordo Moderada- } \\
\text { mente }\end{array}$ & 6 \\
\hline Concordo Fortemente & 7 \\
\hline
\end{tabular}

(b) Itens

\begin{tabular}{|l|}
\hline Itens \\
\hline$I_{1} \cdot$-- Extrovertido, entusiasta. \\
\hline$I_{2} \cdot$-- Crítico, conflituoso. \\
\hline$I_{3} \cdot$-- Confiável, autodisciplinado. \\
\hline$I_{4 \cdot--}$ Ansioso, facilmente perturbável. \\
\hline$I_{5} \cdot$-_ Aberto à novas experiências, complexo. \\
\hline$I_{6} \cdot$-- Reservado, quieto. \\
\hline$I_{7} \cdot$-- Simpático, caloroso. \\
\hline$I_{8} \cdot$-- Desorganizado, sem cuidado. \\
\hline$I_{9 \cdot--}$ Calmo, emocionalmente estável. \\
\hline$I_{10 \cdot--}$ Convencional, sem criatividade. \\
\hline
\end{tabular}

tenha respondido com o valor 6, será mapeado para o valor 2, e assim sucessivamente. Por exemplo, para calcularmos o resultado do usuário no traço Extroversão, utilizamos a resposta dele no primeiro item (Extrovertido, entusiasta) e o reverso da resposta no sexto item (Reservado, quieto). O valor do traço varia de 1 (o traço não é relevante para o usuário) até 7 (o traço é muito relevante para o usuário).

Extrair os traços de personalidade de um indivíduo é uma tarefa complexa. Até o momento, vários trabalhos já vem buscando resolver esse problema de diversas formas, sendo que muitas vezes usam de mecanismos invasivos que demandam informações sensíveis dos usuários. Em relação à extração dos traços de personalidade de usuários de serviços móveis, algumas soluções requerem uma coleta ativa, exigindo um comprometimento do usuário.

Alguns trabalhos encontrados na literatura utilizam a lista de aplicativos ou suas respectivas categorias, e seus respectivos usos, para predizer os traços de personalidade do usuário. [Runhua Xu and Ilic 2016] contabilizam a frequência da utilização das categorias dos aplicativos mais usados entre os usuários para correlacionar com os traços de personalidade através de algoritmos de classificação. Já em [Runhua Xu and Ilic 2015], somente 13 aplicativos das categorias de redes sociais, comunicação, viagens, leitores de textos e compras foram utilizados. O trabalho de [Raihana Ferdous and Mayora 2015] considera somente 5 categorias diferentes para aplicativos e com o objetivo de predizer o nível de estresse no ambiente de trabalho. O presente estudo considera apenas as categorias dos aplicativos instalados pelo usuário, sem a necessidade de mais dados (e.g, frequência de uso), ou seja, com uma única coleta é possível prever os traços de personalidade.

Outros trabalhos utilizam informações de contexto para estimar os traços do usuário, tais como: nível de bateria do celular, quando o dispositivo está carregando, quando o usuário está conectado em uma rede WiFi, quantidade de pessoas com Bluetooth ativado perto do usuário, dentre outros. O trabalho de [Viana et al. 2018] utiliza essas informações para correlacionar com os traços de personalidade. [Gokul Chittaranjan and Gatica-Perez 2011] utilizaram dados dos logs de Bluetooth, SMS e ligações juntamente com as classificações de aplicativos como entrada para 
os classificadores SVM (Support Vector Machine) e C4.5 para prever os traços de personalidade dos usuários. Em [Yves-Alexandre de Montjoye and Pentland 2013], os autores utilizam a quantidade de vezes que o usuário mandou mensagens, realizou ligações e ambos juntos para correlacionar com os traços de personalidade.

Outra base para prever os traços de personalidade é o comportamento do usuário nas redes sociais. [Ryan and Xenos 2011] e [Jianqiang Shen and Liu 2014] baseiam-se no uso do Facebook, como o número de álbuns publicados, as quantidades de compartilhamentos e atualizações do status, para correlacionar com os traços de personalidade. Contudo, para a realização destes estudos são necessárias as coletas de informações privadas dos usuários. Neste trabalho, será utilizada apenas a lista de aplicativos para prever os traços de personalidade dos usuários.

Todos estes trabalhos conseguiram estabelecer uma conexão entre os traços de um indivíduo (personalidade, felicidade, comportamento) e alguma informação contextual de perfil do usuário (social [Doyle and Youn 2000], mobilidade [Viana et al. 2018], uso de dispositivos móveis e aplicativos [Yusong Gao and Zhu 2014]). Esta pesquisa tem como objetivo prever os traços de personalidade do indivíduo levando em consideração apenas as categorias de aplicativos que o mesmo tem instalado em seu smartphone. Diferentemente dos outros trabalhos, a informação sobre as categorias dos aplicativos é mais fácil de obter e menos invasiva em termos de privacidade do usuário. Ou seja, utilizar somente as categorias de aplicativos diminui a complexidade do modelo gerado, facilitando com que o mesmo seja adotado de forma eficiente sem impactar no comportamento do usuário.

\section{Coleta dos Dados}

\subsection{Aplicativo APProfile}

Para obter a base de dados necessária para a análise dos traços de personalidade, foi desenvolvido um aplicativo, denominado APProfile, para dispositivos com sistema operacional Android usando as tecnologias Flutter e Java. Neste aplicativo, o usuário informa sua idade, sexo e responde aos dez itens do formulário TIPI (Tabela 1), e ao final, suas respostas são enviadas para um banco de dados junto com a lista de aplicativos instalados no aparelho móvel do usuário. Por fim, os dados dos usuários são armazenados no Firebase Realtime Database, banco de dados hospedado na nuvem, onde é possível gerar um arquivo JSON contendo todos os dados coletados de cada usuário voluntário. A Figura 1 ilustra duas das telas do aplicativo.

Algumas medidas foram tomadas para impedir ou eliminar possíveis ruídos nos dados. Para evitar que usuários respondessem mais de uma vez, é permitido o reenvio das respostas, porém elas são sobrescritas no banco de dados. Além disso, também foram coletadas a hora em que o usuário começa a responder o questionário e a hora em que envia os dados para verificar a necessidade de remoção de usuários que possam ter respondido aleatoriamente.

O APProfile foi divulgado em algumas redes sociais (Facebook e Instagram) e por e-mail, o tempo da coleta dos dados foi de aproximadamente um mês (entre Maio de 2020 e Junho de 2020), alcançando um total de 98 usuários. Destes usuários, 57 são do sexo masculino e 41 do sexo feminino. A média de idade foi de 22,6 anos, com o desvio padrão igual à 7,15, máximo igual à 63 anos e mínimo igual à 14. Foram considerados 


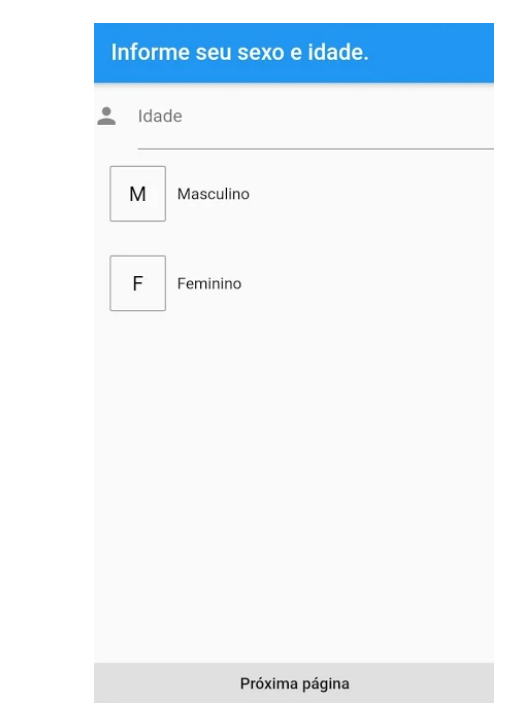

(a) Tela para o preenchimento de Idade e Sexo

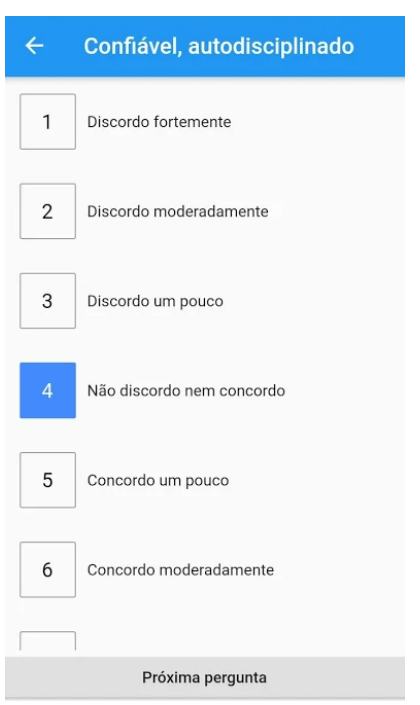

(b) Tela do $\mathrm{Item}_{3}$ do questionário TIPI

Figura 1. Exemplos das telas do APProfile

somente os usuários que levaram pelo menos 45 segundos para responder ao questionário, o que levou ao descarte das respostas de um usuário.

\subsection{A Base de Dados}

Considerando os aplicativos dos 97 usuários, foram coletados dados de 1.423 aplicativos distintos instalados. Foram utilizadas as categorias fornecidas por uma base da Play Store, que permitiu que fossem mapeados 874 aplicativos em 45 categorias distintas. Porém, restaram 549 aplicativos sem categorização, sendo que alguns deles foram instalados por muitos usuários. Para solucionar parcialmente este problema, foi feita manualmente a categorização dos aplicativos que possuíssem pelo menos quatro usuários, totalizando 41 aplicativos. Após a categorização manual, a base continha um total de 915 aplicativos categorizados e 508 aplicativos sem categorização, sendo que esses últimos foram desconsiderados por estarem presentes na lista de poucos usuários. Finalmente, foram removidas as categorias que continham menos de 4 usuários, totalizando 38 categorias. A Figura 2 apresenta a porcentagem de usuários por categoria de aplicativo.

Após a coleta dos dados e o enriquecimento dos aplicativos com suas respectivas categorias, as notas dos traços de personalidade dos usuários foram calculadas. A Figura 3 a mostra a distribuição das notas dos usuários em cada traço de personalidade. A Tabela ?? ilustra um exemplo da matriz completa dos dados apoś todas as agregações e agrupamentos, sendo que os valores das categorias indicam quantos aplicativos daquela categoria um determinado usuário possui, e o valor de cada traço é a nota do usuário para aquele traço específico, que varia de 1 a 7 conforme questionário aplicado.

Existem duas estratégias possíveis para se prever os traços de personalidade: regressão e classificação. Na primeira, o objetivo é inferir a nota do usuário (que varia de 1 a 7) para um traço específico, com base nas categorias dos aplicativos instalados. Já para a estratégia de classificação, é preciso categorizar o usuário em pertencente a um traço caso sua nota seja maior que algum limiar. Neste trabalho, é considerado o limiar de 5,0. Por exemplo, um usuário é considerado Extrovertido (EXT) se sua nota for maior ou igual a 5,0. A Figura 3 b mostra a quantidade de usuários em cada categoria após essa categorização. Vale destacar que um usuário pode pertencer a mais de um traço. 


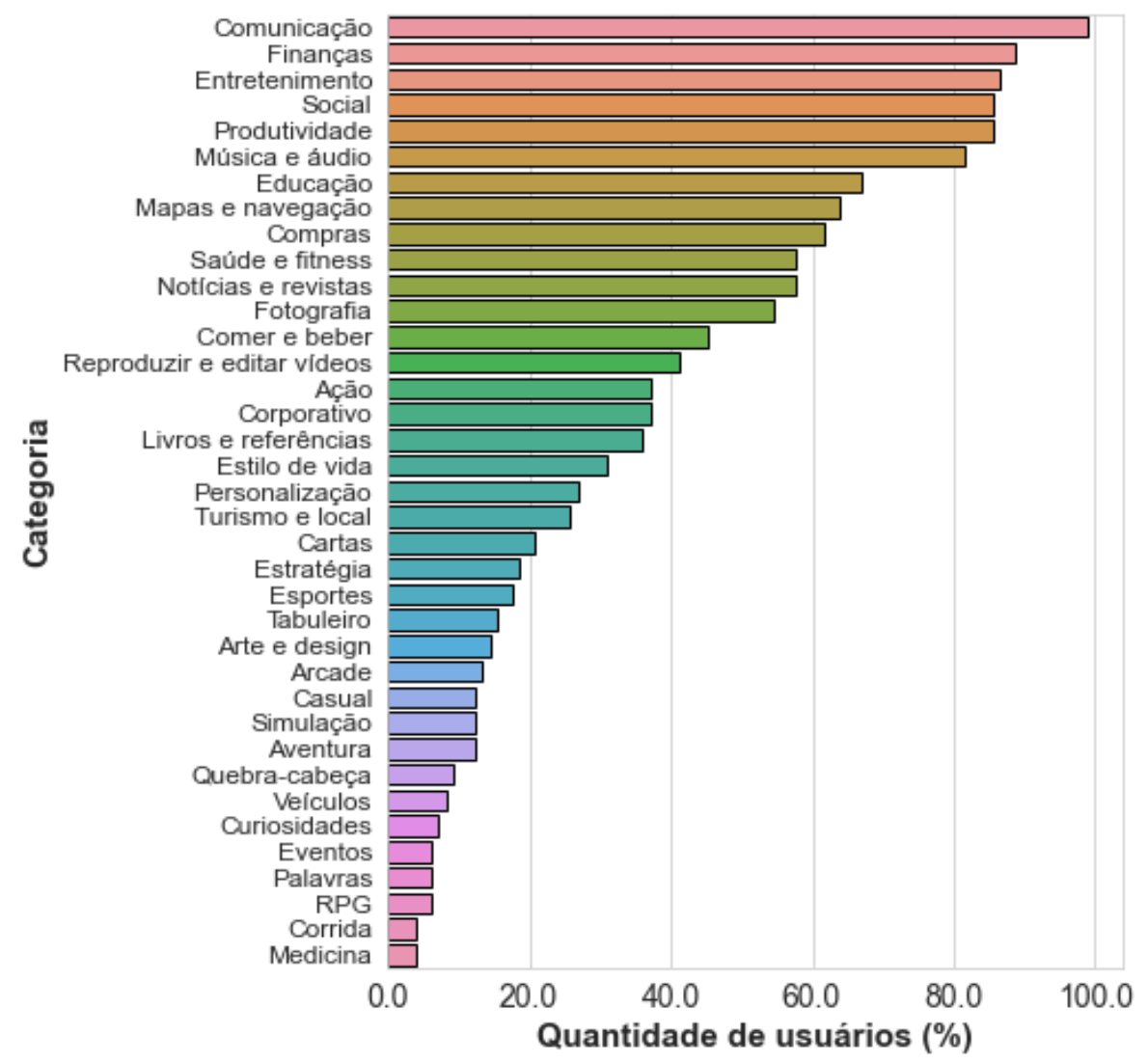

Figura 2. Percentagens de usuários por categoria de aplicativo.

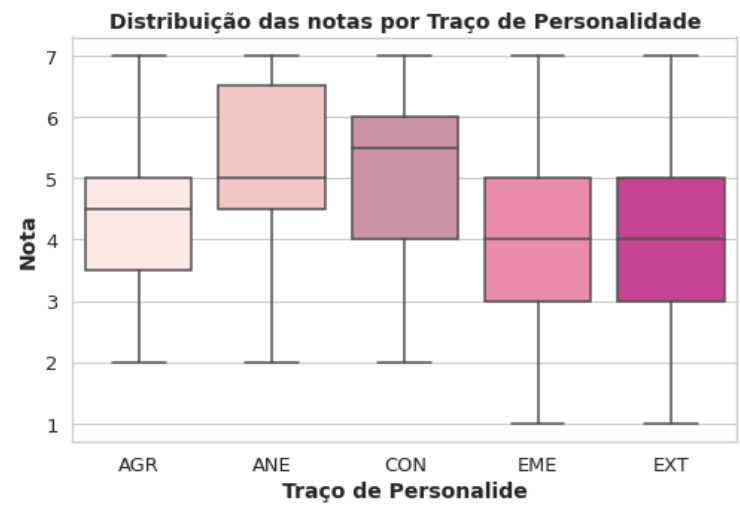

(a) A distribuição dos 5 traços de personalidade.

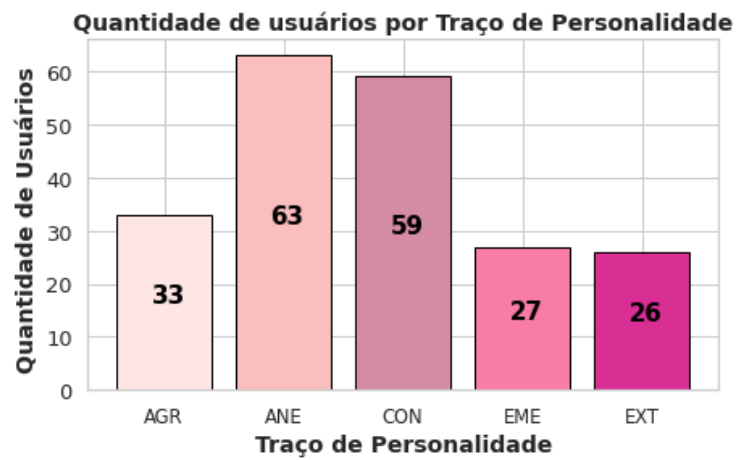

(b) Quantidade de usuários por Traço de Personalidade.

Figura 3. Distribuição das notas (1 a 7) e dos usuários em relação aos traços.

\section{Modelos Preditivos}

Para validar a hipótese do trabalho, foram testadas duas abordagens: classificadores para predizer se o usuário possui ou não o traço de personalidade e modelo de regressão para predizer qual o nível daquele indivíduo em um determinado traço. Antes, foi feita uma análise preliminar para verificar as relações entre categorias de aplicativos e traços de personalidade. 


\subsection{Análise Preliminar}

Para avaliar a relação entre as categorias de aplicativos e os traços de personalidade, foram calculadas as taxas de levantamento (Lift). Nessa taxa, para cada categoria de aplicativo $c$ e traço de personalidade $t$, foram calculadas a percentagem $p 1$ de usuários que possuem $c$ e $t$, em relação a todos os que possuem $t$; e a percentagem $p 2$ de usuários que possuem $c$, mas não o traço de personalidade, em relação a todos os usuários que não possuem o referido traço. Assim, o Lift dessa combinação de categoria e traço de personalidade é dado por $\frac{p 1-p 2}{p 2}$. Dessa maneira, é possível medir o ganho (ou perda, caso negativo) em saber o traço de personalidade do usuário para determinada categoria de aplicativo. A Figura 4 apresenta os 5 maiores e menores Lifts para cada traço de personalidade.

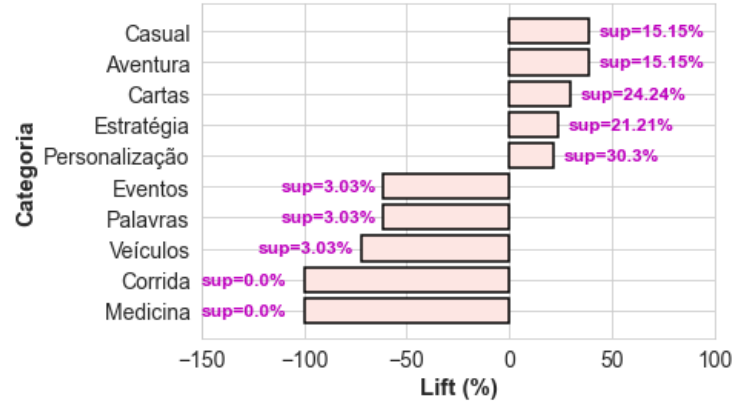

(a) Agradabilidade

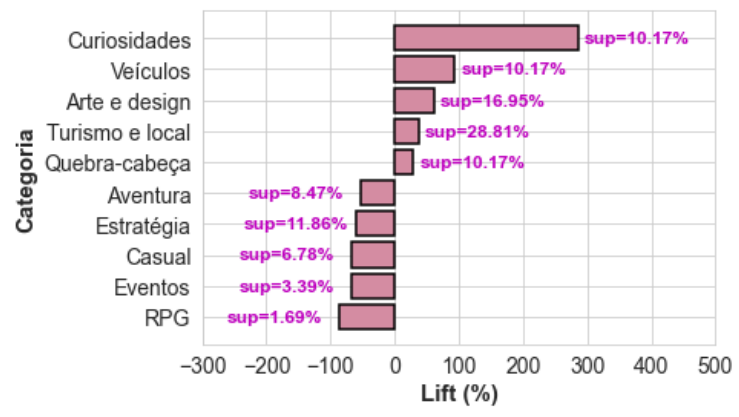

(c) Conscienciosidade

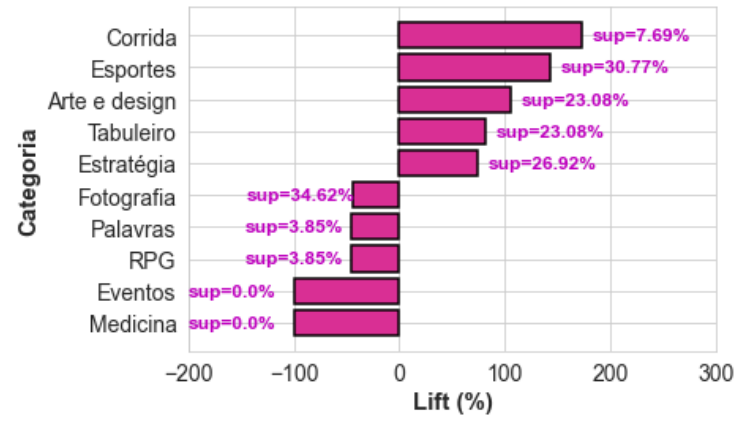

(e) Extroversão

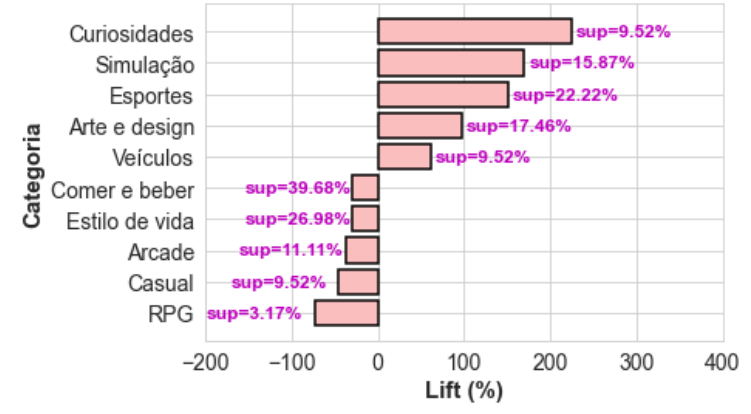

(b) Aberto à Novas Experiências

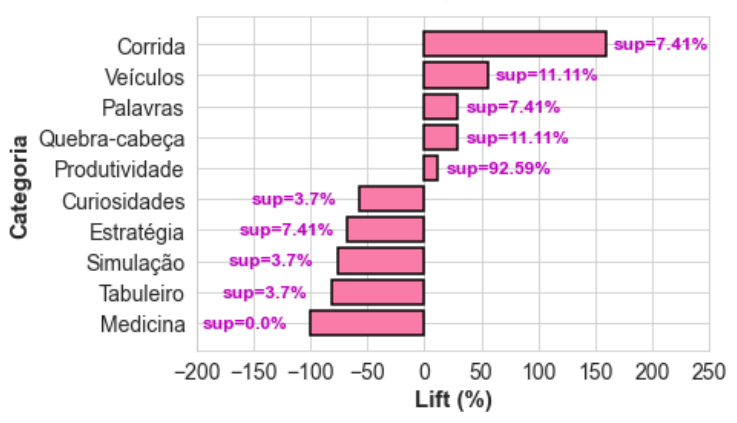

(d) Estabilidade Emocional

Figura 4. Resultados dos Lifts para cada traço de personalidade

A Figura 4a mostra que os maiores Lifts são das categorias Aventura $(38,52 \%)$ e Casual (29,29\%) para usuários que possuem o traço Agradabilidade. Já as categorias com Lifts $-100 \%$ e com suporte $0.0 \%$ indicam que poucos usuários com este traço instalaram aplicativos destas categorias. 
A Figura $4 b$ apresenta Lifts positivos para as categorias Curiosidades e Simulação para usuários que possuem o traço Aberto a Novas Experiências. Estes valores podem estar relacionados ao fato de que pessoas com este traço estão sempre procurando por diferentes experiências. Já com os valores negativos para $R P G(-73,02 \%)$ e Casual ($46,03 \%$ ) não se pode elaborar nenhuma hipótese. A Figura 4c mostra um Lift alto para aplicativos da categoria Curiosidades em relação a usuários com o traço Conscienciosidade (286,44\%). Já o Lift mais baixo para esta classe de personalidade foi para a categoria $R P G(-87,12 \%)$.

Os Lifts mais altos são dos aplicativos das categorias Corrida $(159,26 \%)$ e Veículos $(55,56 \%)$ para o traço Estabilidade Emocional, conforme Figura 4d. Já a categoria Medicina possui Lift $-100 \%$ e suporte $0.0 \%$ indicando que poucos usuários que possuem este traço instalaram aplicativos destas categorias. A Figura $4 \mathrm{e}$ mostra que as categorias que obtiveram os Lifts mais altos são de Corrida $(173,08 \%)$ e Esportes $(142,74 \%)$ para usuários que possuem o traço Extroversão. Os aplicativos das categorias Eventos e Medicina possuem Lifts $-100 \%$ e suporte $0.0 \%$ mostrando que poucos usuários extrovertidos possuem aplicativos destas categorias.

Esses resultados mostram que existem diferenças entre os usuários com diferentes traços em relação aos seus interesses em aplicativos. Portanto, a possibilidade de ser ter modelos preditivos é promissora.

\subsection{Modelos de Classificação}

Para as entradas dos modelos de classificação, foi realizado um mapeamento para os traços de personalidade, sendo 1 caso o usuário possua nota maior ou igual a 5 , e 0 caso contrário. Para corrigir o desbalanceamento da entrada, foi aplicada a técnica de Undersampling, que consiste em pegar amostras do maior grupo e deixá-lo com o mesmo tamanho do grupo menor. Para evitar que o uso desta desta técnica afete os resultados, todo o processo foi repetido 35 vezes para cada combinação de entrada. Além disso, a técnica PCA (Análise de Componentes Principais) foi aplicada para eliminar possíveis categorias que seriam prejudiciais à predição.

Foram criados modelos classificadores binários para cada um dos 5 traços de personalidade. Com o objetivo de encontrar os melhores resultados, foram testados 4 algoritmos diferentes: Regressão Logística, Random Forest, Naive Bayes e K-Nearest Neighbors. A fim de simplificar a discussão, a combinação que utiliza o algoritmo Naive Bayes foi escolhida por possuir, em geral, os melhores resultados de precisão, revocação e FScore. A Figura 5 apresenta os resultados das métricas. A melhor precisão obtida foi no traço Aberto a Novas Experiências, alcançando 100\%. Já para a métrica revocação, o traço Agradabilidade foi o destaque. Ademais, os traços obtiveram, na média, resultados satisfatórios de F-Score, com um pequeno destaque para o traço Agradabilidade.

\subsection{Modelo de Regressão}

Na modelagem da regressão, o método escolhido foi o de Mínimos Quadrados Ordinários (OLS). Foi criado um modelo de regressão para cada traço de personalidade, em que a variável a ser predita é a nota ( 1 a 7) do usuário naquele traço. Para a entrada do modelo, foi considerada a quantidade de aplicativos instalados em cada uma das 38 categorias.

Alguns resultados sobre a regressão podem ser encontrados na Tabela 2 . Pode-se perceber que o $R^{2}$ está acima de 0,81 para todos os traços, o que indica que o modelo 


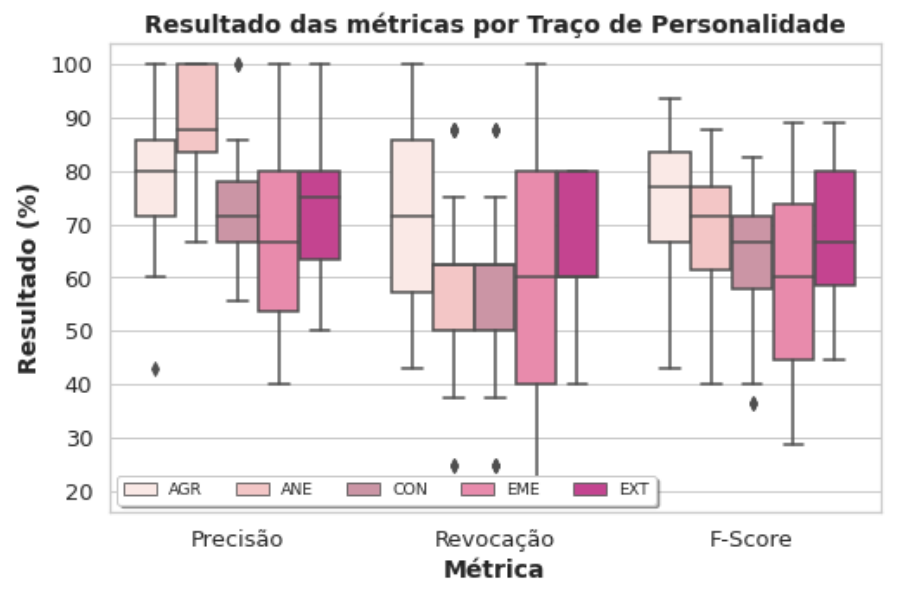

Figura 5. Resultados para Precisão, Revocação e F-Score para os 5 traços.

explica bem a variância da nota de personalidade se comparada com a média. Mesmo quando o $R^{2}$ é ajustado de acordo com o número de variáveis, esse valor ainda é significativo, sendo acima de 0,68 para todos os traços. Por fim, pelos resultados do F-Test, a probabilidade abaixo de 0,05 indica que as variáveis preditoras utilizadas no modelo são relevantes para a estimativa da nota de personalidade com um nível de confiança de $95 \%$.

Tabela 2. Resultado dos modelos de regressão para os traços de personalidade.

\begin{tabular}{ccccc}
\hline Traço & $R^{2}$ & $R^{2}$ Ajus. & F-statistic & Prob (F-statistic) \\
\hline AGR & 0,833 & 0,726 & 7,748 & $2,84 \times 10^{-12}$ \\
\hline ANE & 0,857 & 0,764 & 9,281 & $5,11 \times 10^{-14}$ \\
\hline CON & 0,822 & 0,707 & 7,170 & $1,50 \times 10^{-11}$ \\
\hline EME & 0,811 & 0,689 & 6,664 & $6,95 \times 10^{-11}$ \\
\hline EXT & 0,824 & 0,711 & 7,266 & $1,13 \times 10^{-11}$ \\
\hline
\end{tabular}

Neste trabalho, todos os traços de personalidade se mostraram positivamente associados a aplicativos da categoria Finanças (considerando um nível de confiança de 95\%); isso indica que independentemente do traço, os usuários estão adotando bancos digitais, cartões virtuais e por pagamentos online. Todos os traços de personalidade se mostraram negativamente associados a aplicativos da categoria Estilo de Vida (considerando um nível de confiança de 95\%), que incluem aplicativos de encontros (Tinder, happn, etc.), dieta, dentre outros. O possível motivo disto seria essa época de pandemia, onde as pessoas deixam de utilizar estes aplicativos de encontros e abandonam suas rotinas habituais devido a quarentena.

\section{Conclusão e Trabalhos Futuros}

Este trabalho investigou a possibilidade de predizer os traços de personalidades de um usuário móvel com base nas categorias de seus aplicativos instalados. Os resultados obtidos foram promissores, com bons resultados em termos de precisão, revocação e $F$-Score para a maioria dos traços analisados. Os resultados de uma regressão também foram interessantes, com valores altos de $R^{2}$. Com isso, foi possível mostrar que somente utilizando as categorias dos aplicativos é possível prever os traços de personalidade dos usuários com uma boa assertividade. Como trabalhos futuros, pretende-se levar em consideração as datas em que os aplicativos foram atualizados, para que o perfil do usuário em termos de interesses mais recentes sejam considerados. 


\section{Referências}

Doyle, K. O. and Youn, S. (2000). Exploring the traits of happy people. In Social Indicators Research.

Gokul Chittaranjan, J. B. and Gatica-Perez, D. (2011). Who's who with big-five: Analyzing and classifying personality traits with smartphones. In 15th Annual International Symposium on Wearable Computers, pages 29-36.

Gosling, S. D., Rentfrow, P. J., and Swann Jr, W. B. (2003). A very brief measure of the big-five personality domains. Journal of Research in personality, 37(6):504-528.

Jianqiang Shen, O. B. and Liu, J. (2014). A study of facebook behavior: What does it tell about your neuroticism and extraversion? In Computers in Human Behavior.

M. C. Grace, W. Zhou, X. J. and Sadeghi, A.-R. (2012). Unsafe exposure analysis of mobile in-app advertisements. In 5th conference on Security and Privacy in Wireless and Mobile Networks, pages 101-112.

McCrae, R. R. and Jr., P. T. C. (1999). A five-factor theory of personality. In Handbook of personality: Theory and research, pages 139-153.

Raihana Ferdous, V. O. and Mayora, O. (2015). Smartphone app usage as a predictor of perceived stress levels at workplace. In International Conference on Pervasive Computing Technologies for Healthcare.

Runhua Xu, Remo M. Frey, D. V. and Ilic, A. (2015). Towards understanding the impact of personality traits on mobile app adoption - a scalable approach. In European Conference on Information Systems.

Runhua Xu, Remo Manuel Frey, E. F. and Ilic, A. (2016). Understanding the impact of personality traits on mobile app adoption - insights from a large-scale field study. In Computers in Human Behavior, pages 244-256.

Ryan, T. and Xenos, S. (2011). Who uses facebook? an investigation into the relationship between the big five, shyness, narcissism, loneliness, and facebook usage. In Computers in Human Behavior.

Silva, I. B. and Nakano, T. C. (2011). Modelo dos cinco grandes fatores da personalidade: Análise de pesquisas. In Avaliação Psicológica, pages 51-62.

Viana, A. C., Luzio, A. D., Jaffrès-Runser, K., Stefa, A. M., and Julinda (2018). Accurately inferring personality traits from the use of mobile technology. In HAL Archives Ouvertes.

Yusong Gao, H. L. and Zhu, T. (2014). Predicting subjective well-being by smartphone usage behaviors. In International Conference on Health Informatics.

Yves-Alexandre de Montjoye, Jordi Quoidbach, F. R. and Pentland, A. S. (2013). Predicting personality using novel mobile phone-based metrics. In SBP 2013, pages 48-55. 